\title{
Peripheral and tumor immune correlates in patients with advanced melanoma treated with nivolumab (anti-PD-1, BMS-936558, ONO-4538) monotherapy or in combination with ipilimumab
}

\author{
Michael A Postow ${ }^{1 *}$, Diana M Cardona ${ }^{2}$, Janis M Taube ${ }^{3}$, Robert A Anders ${ }^{3}$, Clive R Taylor ${ }^{4}$, Jedd D Wolchok \\ Margaret K Callahan', Michael A Curran', Alexander M Lesokhin'1, Joseph F Grosso ${ }^{5}$, Christine E Horak', \\ John Cogswell ${ }^{5}$, Jason S Simon ${ }^{5}$, Ashok K Gupta ${ }^{5}$, Mario Sznol ${ }^{6}$
}

From Melanoma Bridge meeting 2013

Naples, Italy. 5-8 December 2013

\section{Background}

The fully human monoclonal antibodies nivolumab (Nivo) and ipilimumab (Ipi) block the interaction between the immune checkpoint receptors programmed death-1 (PD-1) and cytotoxic T lymphocyte antigen-4 (CTLA-4), respectively, and their cognate ligands, restoring antitumor immune response. Phase 1 studies of Nivo monotherapy (CA209-003; NCT00730639) or Nivo+Ipi combination therapy (CA209-004; NCT01024231) demonstrated durable clinical activity and objective response rates (ORRs) of $31 \%$ to $40 \%$, respectively, in patients with advanced melanoma (MEL). Evaluation of PD-L1 expression using immunohistochemistry (IHC) suggested a correlation between pretreatment tumor PD-L1 expression and clinical response to Nivo monotherapy [1]. Identification of predictive markers of response would be valuable to guide effective use of Nivo and Ipi.

\section{Materials and methods}

MEL patients received Nivo monotherapy $(\mathrm{n}=107)$ or Nivo + Ipi combination therapy $(\mathrm{N}=86$; concurrent regimen: $n=53$; sequenced regimen: $n=33$ ). Tumor and tumor infiltrating lymphocyte (TIL) surface programmed death ligand 1 (PD-L1) expression in formalin-fixed, paraffinembedded (FFPE) tumor tissue was evaluated by IHC with an automated assay (Dako) using the 28-8 Ab. Tumor PD-L1 positivity (PD-L1+) was defined as $\geq 5 \%$ cell

* Correspondence: postowm@mskcc.org

${ }^{1}$ Memorial Sloan-Kettering Cancer Center, New York, NY, USA

Full list of author information is available at the end of the article membrane staining of any intensity; any expression on TILs was considered positive. Absolute lymphocyte counts (ALC) were measured in serial peripheral blood samples and lymphocyte subsets were evaluated using flow cytometry.

\section{Results}

Tumor PD-L1-positive expression was observed in 45\% and $37 \%$ of samples from the 003 and 004 studies, respectively. In 003, inclusion of any immune cell staining increased PD-L1 positivity to $92 \%$. A numerically higher ORR was observed in MEL patients with PD-L1+ tumors with Nivo monotherapy or with sequential but not concurrent combination therapy. Neither study demonstrated an obvious change in ALC; however, phenotypic changes in T-cell subsets, including increases in the percentage of CD4 and CD8 expressing HLA-DR, ICOS and/or Ki67, were seen with combination therapy. In both studies, responses were observed irrespective of tumor PD-L1 or ALC status. In an exploratory analysis low pretreatment myeloid derived suppressor cells (MDSC) correlated with higher ORR with combination therapy $(P<0.05)$.

\section{Conclusions}

PD-L1 positivity is associated with tumor response with Nivo monotherapy; however, some responses were observed independent of PD-L1 or ALC status. No correlation between response and PD-L1 or ALC status was seen with combination therapy. MDSC levels may correlate with response to combination therapy. Future 
phase 3 randomized studies will explore these markers and other phenotypic changes in immune cell populations that might predict activity of Nivo in patients with MEL and other advanced cancers.

\section{Authors' details}

${ }^{1}$ Memorial Sloan-Kettering Cancer Center, New York, NY, USA. ${ }^{2}$ Duke University School of Medicine, Durham, NC, USA. ${ }^{3}$ The Sidney Kimmel

Comprehensive Cancer Center at Johns Hopkins, Baltimore, MD, USA. ${ }^{4}$ Keck School of Medicine, University of Southern California, Los Angeles, CA, USA.

${ }^{5}$ Bristol-Myers Squibb, Princeton, NJ, USA. ${ }^{6}$ Yale University School of

Medicine and Yale Cancer Center, New Haven, CT, USA.

Published: 6 May 2014

\section{Reference}

1. Topalian S, et al: N Engl J Med 2012, 366:2443-54.

doi:10.1186/1479-5876-12-S1-08

Cite this article as: Postow et al:: Peripheral and tumor immune

correlates in patients with advanced melanoma treated with nivolumab

(anti-PD-1, BMS-936558, ONO-4538) monotherapy or in combination

with ipilimumab. Journal of Translational Medicine 2014 12(Suppl 1):O8,

\section{Submit your next manuscript to BioMed Central} and take full advantage of:

- Convenient online submission

- Thorough peer review

- No space constraints or color figure charges

- Immediate publication on acceptance

- Inclusion in PubMed, CAS, Scopus and Google Scholar

- Research which is freely available for redistribution

Submit your manuscript at www.biomedcentral.com/submit 